\title{
Bigger DNA: New Double Helix with Expanded Size***
}

\author{
Andreas Marx* and Daniel Summerer
}

\author{
Keywords: \\ DNA . DNA structures - fluorescence . \\ oligonucleotides - supramolecular chemistry
}

\begin{abstract}
Be
esides its immense biological importance, DNA is becoming an increasingly interesting target for chemical modifications. These efforts are driven by numerous motivations, which include therapeutic approaches, functional investigations of enzymes, and new devices for nanotechnology. ${ }^{[1]}$ In the past, new designs of nucleobase scaffolds were achieved by replacing one or more of the natural base pairs by analogues that have different hydrogen-bonding patterns, are isosteric, but with reduced hydrogen-bonding ability, are hydrophobic, or metal-ion binding. ${ }^{[2]}$ All four bases of DNA have never been replaced by artificial scaffolds and it is not clear whether this would be possible without significant loss of its sequence-recognition and spontaneous self-assembly properties.
\end{abstract}

Recently, Kool and co-workers described a new system in which the unmodified DNA backbone is maintained but two of its nucleobases are replaced by extended analogues. ${ }^{[3]}$ The analogues were designed by fusing a benzene ring between the WatsonCrick-recognition sites and the connection of the nucleobase to the sugar moiety. This insertion results in a $2.4 \AA$ size-expansion and converts the bicyclic purine $\mathrm{A}$ into the three-ring system $\mathrm{xA}$ and the cyclic pyrimidine $T$ into bicy-

[*] Priv.-Doz. Dr. A. Marx Dipl.-Chem. D. Summerer Kekulé-Institut für Organische Chemie und Biochemie Universität Bonn Gerhard-Domagk-Strasse 1 53121 Bonn (Germany) Fax: (+49) 228-73-5388 E-mail: a.marx@uni-bonn.de

[***] We thank E. T. Kool, Stanford, for kindly providing the coordinates of DNA and xDNA duplexes. clic $\mathrm{xT}$. Since this transformation does not affect the hydrogen-bonding sites Kool et al. reasoned that artificial sequences containing these bases should form a double helix when combined with DNA containing complementary nucleotide bases. The work was inspired by work of Leonard et al. who used $\mathrm{xA}$ to probe ATP-dependent enzymes three decades ago ${ }^{[4]}$ From the modified bases $\mathrm{xA}$ and $\mathrm{xT}$ Kool et al. synthesized the nucleoside analogues dxA and dxT and
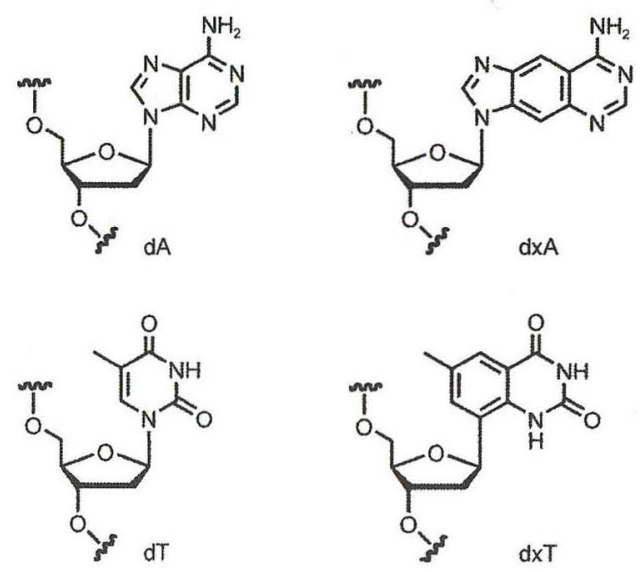

were able to incorporate them into oligonucleotides and study their properties.

To elucidate whether the extended analogues form Watson-Crick base pairs in an unmodified-DNA environment Kool et al. investigated duplexes containing single extended bases (xA or $\mathrm{xT})$ near the middle of a 12-base-pair oligonucleotide (1, 2 in Figure 1). They found that both analogues when paired to the unmodified partner destabilize the double helix relative to the unmodified double helix. Through investigation of nucleobase variations opposite the extended analogues it became apparent that $\mathrm{xA}$ has a slight pairing selectivity for $\mathrm{T}$ over $\mathrm{C}, \mathrm{G}$, or $\mathrm{A}$ and that $\mathrm{xT}$ in $\mathbf{2}$ is more unselective in pairing to its partner in the opposite strand of the duplex. These observations indicate that the DNA backbone is too rigid to tolerate alterations, since significant distortion of the helical conformation would be re-

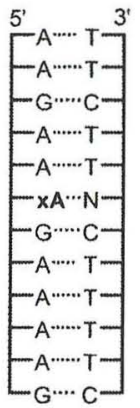

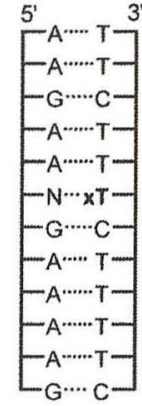

2

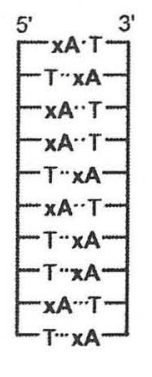

3

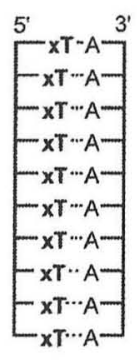

4

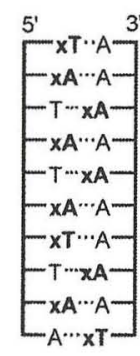

5
Figure 1. Selected DNA and extended DNA (xDNA) constructs. ${ }^{[3]}$ All xDNA structures were compared with corresponding DNA structures containing exclusively natural building blocks. Constructs 1 and 2 exhibit decreased and 3-5 increased duplex stability when compared with the structures made up of natural building blocks. 
quired to accommodate the extended base analogues within a regular DNA helix.

Kool et al. speculated that a helix exclusively composed out of the extended nucleotides would circumvent the geometric constraints dictated by the regular DNA helix. Thus, a self-complimentary oligonucleotide $\mathbf{3}$ was investigated that formed a helix with an expanded nucleotide in every single base pair. They found that this $\mathrm{xA}$ residue containing complex $\mathbf{3}$ is more stable than the natural duplex by $5.8 \mathrm{kcal}$ $\mathrm{mol}^{-1}$. To test the sequence generality of the system several other duplexes were investigated. Consistent with the above results, increased stability was found for double helixes with other sequences but in which every base pair included an extended base (4 and $\mathbf{5}$, Figure 1). What is the origin of the increased stability of the enlarged xDNA helix? Kool et al. ascribe the measured increase in pairing stability to enhanced stacking of the enlarged aromatic systems. Stacking might occur either within each strand or across the strands. NMR spectroscopy investigations corroborated that a duplex system with significant interstrand hydrogen bonding is indeed formed in cases where size-expanded analogues were used. A self-complementary sequence was investigated and found to give rise to the number of NMR resonance signals consistent with a antiparallel duplex structure. Modeling studies provided insights into the structural differences between normal B-form DNA and xDNA (Figure 2).

Structural and thermodynamic investigations indicate only small conformational changes in the DNA sugarphosphate backbone and suggest that the larger diameter of the xDNA helix results in a greater number of bases per

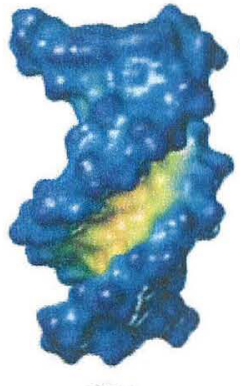

DNA

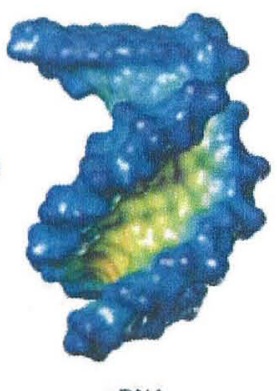

xDNA
Figure 2. Side view of 10-base-pair DNA and modeled xDNA duplexes. Shown are the Connolly surfaces with cavity depth-dependent coloring (blue shallow, green deep). The structures were generated with the SYBYL6.9 (TRIPOS) software package.

turn than in B-DNA. Additionally, size extension might cause the minor and major grooves of the xDNA-helix to be wider than those of B-DNA.

The development of xDNA is an outstanding and very elegant example for a successful molecular design. The report by Kool et al. points once again at the great potential of nucleic acids as suitable scaffolds for nanoscale engineering. ${ }^{[1]}$ The enlarged oligonucleotides harbor features not found in natural DNA: Owing to the incorporation of the fluorescent monomers $\mathrm{xA}$ and $\mathrm{xT}$ the nucleotides $\mathrm{dxA}$ and $\mathrm{dxT}$ are highly fluorescent, as are the resulting oligonucleotides. Thus, in xDNA all the base pairs are fluorescent giving a polymer with high fluorophore density. Kool et al. speculate that this property of xDNA combined with its increased binding propensity might be particularly useful for applications in detection and diagnosis of DNA or RNA. For this purpose as well as to complete the sizeextended genetic alphabet, the to-date missing nucleobase surrogates $\mathrm{xG}$ and $\mathrm{xC}$ are awaited. Their preparation might enable further size-expansion, for example, through construction of a helix composed exclusively of size-extended nucleotides. A further investigation would be to see if DNA polymerases process the extended analogues. As noted by Kool recently, ${ }^{[5]}$ these analogues might be useful to test the hypothesis of "active-site tightness" for DNA polymerases. This model is proposed as an explanation for enzyme selectivity and its variation among several DNA polymerases. ${ }^{[5]}$ We await fascinating progress along these lines in near future.

[1] Reviews: a) A. DeMesmaeker, R. Häner, P. Martin, H. Moser, Acc. Chem. Res. 1995, 28, 366-374; b) Y. S. Sanghvi in DNA and Aspects of Molecular Biology (Ed.: E. T. Kool), Pergamon, Oxford, 2002, chap. 8 ; c) S. Verma, F. Eckstein, Annu. Rev. Biochem. 1998, 67, 99-134; d) L. W. McLaughlin, M. Wilson in DNA and Aspects of Molecular Biology (Ed.: E. T. Kool), Pergamon, Oxford, 2002 chap. 7; e) N. C. Seeman, Nature 2003, $421,427-431$; f) C. M. Niemeyer, M. Adler, Angew. Chem. 2002, 114, $3933-$ 3937; Angew. Chem. Int. Ed. 2002, 41, 3779-3783; g) A. Marx, I. Detmer, J. Gaster, D. Summerer, Synthesis 2004, 1 14.

[2] Reviews: a) E. T. Kool, J. C. Morales, K. M. Guckian, Angew. Chem. 2000, 112, 1046-1968; Angew. Chem. Int. Ed. 2000, 39, 990-1009; b) E. T. Kool, Acc. Chem. Res. 2002, 35, 936-943; c) H.-A. Wagenknecht, Angew. Chem. 2003, 115, 3322-3324; Angew. Chem. Int. Ed. 2003, 42, 3204-3206.

[3] H. Liu, J. Gao, S. R. Lynch, Y. D. Saito, L. Maynard, E. T. Kool, Science 2003, 302, 868-871.

[4] a) N. J. Leonard, M. A. Sprecker, A. G. Morrice, J. Am. Chem. Soc. 1976, 98 , 3987; b) R. A. Lessor, K. J. Gibson, N. J. Leonard, Biochemistry 1984, 23, 3868.

[5] E. T. Kool, Annu. Rev. Biochem. 2002, 71 , $191-219$. 